\title{
Primary nursing: a Varese un Progetto sinergico tra infermieri e medici per aumentare l'adesione terapeutica dei pazienti nefropatici
}

\author{
Laura Bardelli ${ }^{1}$, Stefania Bordiga ${ }^{2}$, Roberta Foglia ${ }^{2}$ \\ ${ }^{1}$ Coordinatore Infermieristico, S.C. Nefrologia e Dialisi, ASST dei Sette Laghi, Varese - Italy \\ ${ }^{2}$ Infermiera, S.C. Nefrologia e Dialisi, ASST dei Sette Laghi, Varese - Italy
}

\begin{abstract}
Primary nursing: in Varese a synergistic project between nurses and doctors to increase the therapeutic adhesion of renal patients

The World Health Organization (WHO) defines therapeutic adherence as "the extent to which a patient's behavior - in taking medications, following a diet and/or making lifestyle changes - corresponds to the recommendations of health professionals in charge".

Chronic disease is permanent and requires by the patient an active attitude to reach and maintain a state of wellbeing, and to be often subject to long periods of supervision, observation and care.

Trust must therefore be established between patient and healthcare professionals.

This is the reason why it is not correct to discuss compliance. The main difference is that therapeutic adherence requires the patient's agreement with the prescriptions: patients should be an active partner in their own care and communication between the patient and healthcare professionals is required for an efficient clinical practice.
\end{abstract}

Keywords: Chronic disease, Compliance, Nursing, Therapy

La probabilità di aderire a un programma terapeutico è tanto più elevata quanto più quel programma è semplice. Non solo: anche a fronte di una terapia semplice, il doverla assumere per numerosi anni riduce di molto la probabilità di assumere correttamente la terapia. Lo stesso succede quando si deve curare in modo preventivo una malattia di cui non si ha la percezione concreta dei danni: anche in questo caso l'adesione è piuttosto scarsa.

Negli ultimi anni questo argomento è diventato particolarmente importante, perché i pazienti sono sempre più complessi e spesso devono curare più patologie insieme, di conseguenza assumono molti più farmaci.

$\mathrm{Ci}$ sono numerosi studi che documentano come almeno il $50 \%$ dei pazienti diabetici non segua correttamente la terapia, e questa osservazione vale per molte patologie. Per

Received: October 30, 2020

Accepted: November 3, 2020

Published online: December 7, 2020

Indirizzo per la corrispondenza:

Laura Bardelli

Servizio Dialisi

ASST dei Sette Laghi

Viale Borri, 57

21100 Varese - Italy

laura.bardelli@asst-settelaghi.it esempio, è riconosciuto che, nei pazienti che hanno avuto un evento cardiaco acuto, la non adesione alla terapia comporta un rischio di morte di circa il 15\%. Malgrado ciò, l'adesione al programma terapeutico non è quasi mai ottimale (1).

La Figura 1 documenta in modo evidente come il dover assumere farmaci una, due, tre o quattro volte al giorno riduca drasticamente l'adesione alla terapia.

Chiaramente $\mathrm{i}$ farmaci prescritti che non si assumono costano e non sono efficaci. Una categoria di pazienti particolarmente esposta a questo problema è rappresentata dai pazienti nefropatici durante tutte le fasi della malattia, cioè sia prima di cominciare la dialisi che durante e dopo il trapianto.

Le ragioni per questa scarsa aderenza alla terapia sono molteplici ma, a differenza di quanto in prima ipotesi si possa pensare, non dipendono solo dalla numerosità delle compresse. Le cause più importanti sembrano essere: la motivazione e la condivisione del programma (2-3).

Volutamente, fin a questo punto, abbiamo parlato di adesione al programma terapeutico, che presuppone che il paziente debba aderire passivamente a quanto stabilito dal medico.

È evidente che, se il paziente non condivide (comprende) la complessità e le molte sfaccettature delle sue patologie, non vive l'assunzione della terapia come qualcosa che realmente lo aiuti e possa farlo vivere meglio. 


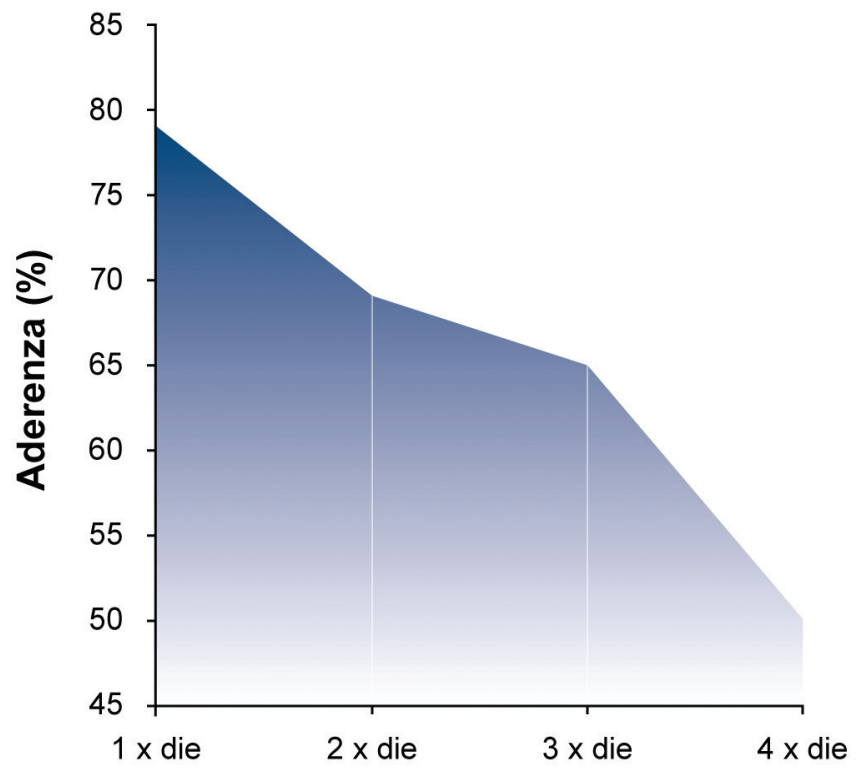

Piano terapeutico

Fig. 1 - Riduzione dell'aderenza al piano terapeutico in base alla frequenza di somministrazione.

L'Organizzazione Mondiale della Sanità nel 2003 ha proposto di indagare su alcuni settori del processo adesione/ assunzione/consapevolezza, per comprenderlo meglio e, quindi, migliorare l'adesione alla terapia.
La WHO propone i seguenti settori d'indagine:

Fattori legati al sistema sanitario e al team assistenziale Fattori legati al territorio

Fattori socio-economici

Fattori legati alla terapia

Fattori legati al paziente

Molti centri dialisi, soprattutto all'estero, hanno cominciato a porsi questo problema alcuni anni fa e hanno elaborato una griglia per valutare la consapevolezza della terapia mediante uno schema visivo degli orari (Fig. 2).

Gli autori lo considerano efficace, anche se non ottimale. II problema principale di questa discrepanza sta nel fatto che la prescrizione terapeutica la fa il medico, mentre la verifica è affidata all'infermiere, che non sempre partecipa al processo prescrittivo. In Italia, infatti, gli infermieri non possono prescrivere farmaci né esami.

In molti paesi stranieri questo è possibile da numerosi anni. Negli Stati Uniti, gli infermieri sono parte attiva nelle prescrizioni già dal 1960, mentre il primo paese europeo a introdurre questa nuova modalità è stato la Svezia nel 1994, seguita da Spagna e Olanda nel 2006 e Irlanda nel 2007. In questo contesto, paziente e infermiere diventano parte attiva del processo, con un continuo confronto fra i due e il medico. Inoltre, c'è un controllo continuo della coerenza dietetica e terapeutica e si ottimizzano le scelte. In pratica, dove infermiere e paziente erano esclusi dalla decisione e dalla scelta,

\begin{tabular}{|c|c|c|c|c|c|}
\hline \multicolumn{6}{|c|}{$\begin{array}{l}\text { Tenere questa tabella aggiornata e portarla con sé ad ogni controllo medico, in farmacia e anche per accessi al Pronto Soccorso } \\
\text { Inserire tutti i farmaci assunti, compresi integratori e farmaci da banco } \\
\text { Evitare di rimanere senza scorte di farmaci, chiedere al medico la prescrizione adeguata }\end{array}$} \\
\hline \multirow{2}{*}{ Farmaco e dosaggio } & \multirow{2}{*}{ Serve per... } & \multicolumn{4}{|c|}{ Quando e quanto viene assunto? } \\
\hline & & $\underbrace{\prime \prime \prime \prime}$ mattina & 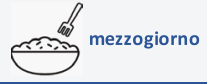 & $\stackrel{111}{\infty \cdots}$ sera & Prima di andare a letto \\
\hline & & & & & \\
\hline & & & & & \\
\hline & & & & & \\
\hline & & & & & \\
\hline & & & & & \\
\hline & & & & & \\
\hline & & & & & \\
\hline & & & & & \\
\hline & & & & & \\
\hline & & & & & \\
\hline & & & & & \\
\hline & & & & & \\
\hline \multicolumn{6}{|c|}{ Per qualsiasi problema con i propri farmaci non aspettare: consultare al più presto medico, infermiere o farmacista } \\
\hline Paziente & & Infermiere & & Farmacista & \\
\hline Nome e cognome & & Nome e cognome & & Nome e cognome & \\
\hline Recapito telefonico & & Recapito telefonico & & Recapito telefonico & \\
\hline
\end{tabular}

Fig. 2 - Esempio di schema terapeutico utilizzato dai pazienti. 
acquistano autonomia e incidono nel processo decisionale da alleati.

È evidente che, per portare avanti un programma di miglioramento del progetto terapeutico, serve un'organizzazione che preveda un ruolo attivo dell'infermiere in ogni fase assistenziale e che aiuti il paziente nelle fasi assistenziali più difficili, compresa la scelta della strategia dialitica (4).

Noi, nei centri dialisi "territoriali" della ASST Settelaghi, stiamo sviluppando e implementando l'adozione di un modello organizzativo definito del Primary Nursing.

Si tratta di un modello olistico, basato sulle relazioni, che concepisce l'assistenza come un servizio alla persona nella sua globalità, quindi in aggiunta ai consueti interventi terapeutici e di sostegno. Per completare e dettagliare l'assistenza, per ogni tematica associata al trattamento dialitico è stato sviluppato uno specifico settore di intervento, ad opera di un gruppo di infermieri dedicati.

Alcuni tra i gruppi implementati fino a oggi: monitoraggio degli accessi vascolari e dell'adeguatezza dialitica, gestione dell'iter diagnostico per l'inserimento/mantenimento in lista trapianto, personalizzazione del follow-up periodico, alleanza terapeutica e presa in carico. Ciascun gruppo è diretto da un'Infermiera primaria (così definita dal modello organizzativo) con provate conoscenze, capacità e motivazione, affiancata da un piccolo numero di colleghi nel ruolo di collaboratori.

La pratica quotidiana di questo modello organizzativo permette al personale infermieristico di accumulare un corpo crescente di conoscenze, strategie, procedure, relazioni e comportamenti, tali da conferire loro la potenzialità per la gestione in autonomia del dializzato, e di assumere piene responsabilità e consapevolezza delle proprie decisioni. Ovviamente, le decisioni vengono sempre condivise con il medico.

Questa gestione partecipata, applicata in ogni settore di intervento infermieristico, potrebbe aprire, un domani, la strada alla possibilità, per gli infermieri, anche di cogestire con il medico farmaci ed esami.

È evidente che, nell'ambito delle patologie croniche dove si sta sviluppando la cultura di spostare l'epicentro dell'azione assistenziale sul territorio e, in particolare, nell'assistenza ai pazienti dializzati, l'alleanza paziente-infermiere è un prerequisito per il pieno successo di questa svolta culturale.

\section{Disclosures}

Conflict of interest: The authors declare no conflict of interest. Financial support: This research received no specific grant from any funding agency in the public, commercial, or not-for-profit sectors.

\section{Bibliografia}

1. Russell S, Daly J, Hughes E, Hoog Co Co. Nurses and 'difficult' patients: negotiating non-compliance. J Adv Nurs. 2003 Aug; 43(3):281-287. Medline CrossRef

2. Martin LR, Williams SL, Haskard KB, DiMatteo RM. The challenge of patient adherence. Ther Clin Risk Manag. 2005;1(3):189-199. Medline

3. Cameron C. Patient compliance: recognition of factors involved and suggestions for promoting compliance with therapeutic regimens. J Adv Nurs. 1996;24:244-250. Medline CrossRef

4. Viswanathan $M$, Golin $C E$, Jones $C D$, et al. Interventions to Improve Adherence to Self-administered Medications for Chronic Diseases in the United States. A Systematic Review. Ann Intern Med. 2012;157:785-795. Medline CrossRef 